\title{
Evaluación neurofisiológica y estructural del comportamiento de la vía visual en pacientes con esclerosis múltiple
}

\section{Neurophysiological and structural evaluation of the behavior of the visual pathway in patients with multiple sclerosis}

\author{
José David Jiménez Mirabal,* Eduardo Perusquía Ortega, ${ }^{\ddagger}$ Antonio De León Aguirre, ${ }^{\S}$
} Iveth Marlem Montoya Aranda, $\$$ Gerónimo Pacheco Aispuro, ${ }^{\S}$ Rafael Arias Flores॥

\section{Resumen}

La evaluación de la vía visual es importante en pacientes con esclerosis múltiple (EM), la tomografía de coherencia óptica (OCT) permite ver in vivo la retina, los potenciales evocados visuales (PEV) son el estudio neurofisiológico más empleado en sospecha de neuritis óptica (NO). Objetivo: Determinar la correlación de estas herramientas con el comportamiento de la enfermedad en pacientes mexicanos con EM. Material y métodos: Se incluyeron 59 pacientes (118 ojos) con esclerosis múltiple (EM) que cumplieron criterios de inclusión: > 18 años, fenotipo EM-RR con EDSS < 5.5 en tratamiento con algún modificador de la enfermedad. A los pacientes se les realizó: valoración neurológica completa con EDSS, examen oftalmológico, PEV convencionales, OCT, IRM cerebral con gadolinio. Resultados: El sexo femenino fue el más frecuente $66.1 \%$, 38 ojos presentaban historia de NO (EM-NO). En los PEVs la onda P-100 con la latencia más prolongada estuvo en $177 \mathrm{~ms}$. $50.85 \%$ tenían espesor G-CFNR reducido, correspondiendo $51.66 \%$ al OI. Se encontraron valores disminuidos de G-CFNR en la OCT basal en pacientes con EM-NO $(p<0.0003)$. Conclu-

\section{Abstract}

Clinical assessment of the visual pathway is an important aspect in patients with multiple sclerosis (MS), visual evoked potentials (VEP) are the most commonly used neurophysiological study when optic neuritis (ON) is suspected. Objective: To determine the correlation of these tools with the behavior of the disease in Mexican patients with MS. Material and methods: 59 patients (118 eyes) with multiple sclerosis (MS) were included, who met the inclusion criteria: > 18 years, EM-RR phenotype with EDSS $<5.5$, in treatment with some disease modifier. To all patients were performed; Complete neurological evaluation with EDSS, ophthalmological examination, conventional VEP, OCT, cerebral MRI with gadolinium. Results: The female sex was the most frequent $66.1 \%, 38$ eyes had a history of ON (EM-ON). In VEPs, the $\mathrm{P}-100$ wave with the longest latency was $177 \mathrm{msec}$. $50.85 \%$ had an average reduction of G-RNFL, $51.66 \%$ corresponding to the LE. Decreased values of G-RNFL were found at baseline OCT in patients with MS-ON ( $p<0.0003)$. Conclusion: These results confirm the sensitivity of PEVs in patients
* Neurólogo, Alumno del Curso de Alta Especialidad en Neurofisiología Clínica. Facultad Mexicana de Medicina, Universidad La Salle. Ciudad de México, México.

₹ Neurofisiólogo Clínico, Hospital Ángeles Pedregal. Profesor Titular del Curso de Alta Especialidad en Neurofisiología Clínica. Facultad Mexicana de Medicina, Universidad La Salle. Ciudad de México, México.

$\S$ Neurofisiólogo Clínico, Hospital Ángeles Pedregal. Profesor adjunto del Curso de Alta Especialidad en Neurofisiología Clínica. Facultad Mexicana de Medicina, Universidad La Salle. Ciudad de México, México.

\| Médico Epidemiólogo, División de Epidemiología Hospitalaria. Unidad Médica de Alta Especialidad, CMN Siglo XXI. IMSS.

Correspondencia:

Dr. José David Jiménez Mirabal

Correo electrónico: dr.jdavidjm@gmail.com

Aceptado: 19-11-2019.

\section{Siglas:}

$\mathrm{EM}=$ Esclerosis múltiple.

$\mathrm{PEV}=$ Potenciales evocados visuales

OCT $=$ Tomografía de coherencia óptica.

$\mathrm{NO}=$ Neuritis óptica

CIS = Síndrome clínico aislado

EDSS = Escala del estado de discapacidad ampliada

CFNR $=$ Capas de fibras nerviosas de la retina

IRM = Imagen de resonancia magnética

TCE $=$ Traumatismo craneoencefálico

$\mathrm{EM}-\mathrm{RR}=$ Esclerosis Multiple Remitente Recurrente.

\section{Abbreviations:}

MS = Multiple sclerosis

VEP $=$ Visual evoked potentials.

OCT $=$ Optical coherence tomography.

www.medigraphic.com/actamedica

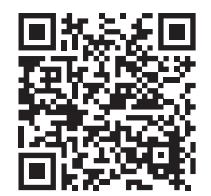


sión: Estos resultados confirman la sensibilidad de los PEVs en pacientes con EM-NO y EM-NON, y el incremento de ésta para detectar daño en la vía visual al utilizar ambos estudios.

Palabras clave: Esclerosis múltiple, potenciales evocados visuales, latencia P-100, tomografía de coherencia óptica, espesor G-CFNR.

\section{INTRODUCCIÓN}

La valoración clínica de la vía visual es un aspecto muy importante en la evaluación de pacientes con diagnóstico definitivo de esclerosis múltiple (EM) o síndrome clínico aislado (CIS), por el hecho de que las alteraciones visuales suelen ser frecuentemente incapacitantes, Ilegando a afectar hasta $80 \%$ de los pacientes, sin olvidar que $50 \%$ pueden presentar neuritis óptica (NO) en algún momento de su vida, siendo entre 15 y $20 \%$ la manifestación inicial de la EM. ${ }^{1,2}$ De ahí nace la importancia de realizar una evaluación funcional y estructural de la vía visual aferente en estos pacientes.

La evaluación funcional en pacientes con EM se lleva a cabo de forma clínica mediante la exploración de la agudeza visual y visión de colores al realizar la revisión por sistema funcional de Kurtzke y la escala de estado de discapacidad ampliada (EDSS), también mediante estudios campimétricos y pruebas electrofisiológicas.

Los potenciales evocados visuales (PEV) son el estudio neurofisiológico más comúnmente empleado hasta el momento para medir la funcionabilidad de la vía visual aferente. Los PEV convencionales miden la respuesta cortical a la estimulación monocular a los $30^{\circ}$ centrales del campo visual, representando la vía visual desde la retina a lo largo del nervio óptico, el quiasma, el tracto y la radiación óptica hacia la corteza occipital. ${ }^{3}$ En la EM, los pacientes con síntomas visuales o historia de NO, la latencia de la onda P-100 se retrasa de manera característica con una amplitud normal, lo que sugiere desmielinización; sin embargo, la pérdida axonal puede disminuir también la amplitud de esta onda. ${ }^{4}$

En pacientes con EM sin historia de $\mathrm{NO}$ se ha encontrado que los PEVs proporcionan evidencia de lesiones clínicamente silenciosas en el nervio óptico que podrían ayudar a identificar diseminación en espacio, ayudando a establecer el diagnóstico y a dar un mejor seguimiento de la actividad de la enfermedad. Esto hace que los PEVs sean una herramienta muy sensible, aunque poco específica para el diagnóstico de neuritis óptica (NO). ${ }^{5}$

La evaluación estructural se puede realizar mediante el campo visual y la tomografía de coherencia óptica (OCT), aunque ésta sólo estudia el nervio óptico en la retina. with MS-ON and EM-NON, as well as the increase of this to detect damage in the visual pathway when using both studies.

Keywords: Multiple sclerosis, visual evoked potentials, P-100 latency, optical coherence tomography, G-RNFL thickness.

La OCT es un método no invasivo que tiene un principio similar al ultrasonido, pero en lugar de ondas acústicas utiliza ondas de luz de alta resolución que nos brinda cortes transversales de la retina, lo que nos permite analizar la cabeza del nervio óptico y el volumen macular en tiempo real, y medir en vivo las capas de fibras nerviosas de la retina $(\mathrm{CFNR})^{6}$ con una alta resolución y reproducibilidad. ${ }^{7}$

Se considera que el adelgazamiento de estas capas (CFNR) se correlaciona con la degeneración retrógrada después del daño al nervio o al tracto óptico en el cerebro, lo que se ha correlacionado con atrofia cerebral, mayor discapacidad funcional en general y deterioro cognitivo, ${ }^{8-10}$ por lo que ha sido propuesto como un biomarcador de actividad de la enfermedad.

De ahí la importancia de conocer y obtener datos sobre el comportamiento electrofisiológico y estructural del nervio óptico en pacientes mexicanos con EM con o sin historia de NO, analizar cómo se correlacionan estos estudios entre ellos en cuanto a la integridad de la vía visual, la actividad en general de la enfermedad, la neurodegeneración desmielinizante y axonal, y su relación con el estado funcional del paciente, por lo que el objetivo de este estudio es determinar la correlación de estas herramientas con el comportamiento de la enfermedad en pacientes mexicanos con EM.

\section{MATERIAL Y MÉTODOS}

\section{Pacientes}

El estudio incluyó 59 pacientes (118 ojos) consecutivos con diagnóstico de esclerosis múltiple (EM), referidos por varios neurólogos de nuestro hospital. El estudio tuvo una duración de 24 meses y se diseñó de acuerdo con los principios de la Declaración de Helsinki para la Investigación Biomédica, obteniéndose el consentimiento informado de todos los participantes.

Los criterios de inclusión fueron: 1. Edad > 18 años, 2. Fenotipo EM-RR con escala del estado de discapacidad ampliada (EDSS) $<5.5$, 3. En tratamiento con algún modificador de la enfermedad durante todo el estudio con adecuado seguimiento y apego terapéutico.

Criterios de exclusión: 1. NO reciente ( $<$ tres meses), 2. En tratamiento con algún medicamento que pudiera produ- 
cir toxicidad al nervio óptico o la retina, y 3 . Antecedentes oftalmológicos de glaucoma, ambliopía o retinopatía.

Criterios de eliminación: 1. Mal apego al tratamiento modificador de la enfermedad $y / o$ al seguimiento en sus citas control cada tres meses.

Recolección de la información

En todos los pacientes se realizó: 1) historia clínica y tiempo de duración de la enfermedad, 2) valoración neurológica completa y por sistemas funcionales de Kurtzke con EDSS, 3) examen oftalmológico con fondo de ojo, agudeza visual lejana, pruebas de visión de colores (test de Ishihara) y campimetría por confrontación, prestando especial atención en antecedentes de alguna alteración visual, historia de neuritis óptica (NO) o hallazgos en el fondo de ojo sugestivos de NO, 4) PEV convencionales, 5) OCT con medición promedio del espesor de CFNR peripapilar, 6) IRM cerebral con gadolinio, 7) exámenes generales de laboratorio $(\mathrm{BH}, \mathrm{QS}, \mathrm{ES}, \mathrm{PFH}$, perfil de lípidos y pruebas de coagulación). Estos estudios se realizaron el mismo día de la valoración neurológica inicial.

Se realizaron evaluaciones neurológicas completas con escala de discapacidad funcional por sistemas de Kurtzke y EDSS cada tres meses, IRM con gadolinio cada 12 meses y al concluir los 24 meses se realizó nuevamente OCT a los 42 pacientes que continuaban en el estudio, ya que 17 pacientes salieron del mismo; 16 por cumplir criterios de eliminación y un paciente falleció por razones ajenas a la EM (TCE severo).

\section{Potenciales evocadas visuales (PEVs) convencionales}

Los PEVs se realizaron con un equipo Viasys/Nicolet Viking Quest de cuatro canales, versión 20.1.13. utilizando el tipo de registro con estimulador LED flash estroboscópico mediante goggles. En todos los pacientes se midieron las latencias de la onda P-100 así como la morfología y amplitud de esta respuesta al inicio del estudio, todas las mediciones fueron evaluadas por dos neurólogos expertos. La latencia de la onda P-100 fue considerada prolongada a partir de $120 \mathrm{~ms}$.

\section{Tomografía coherencia óptica (OCT)}

La tomografía de coherencia óptica (OCT) se realizó con un equipo Optovue RTVue System. Se midió el espesor de las capas de fibras nerviosas de la retina (CFNR) peripapilar en los cuatro cuadrantes (temporal, nasal, inferior y superior) así como el promedio de forma global (G-CFNR) al inicio del estudio y a los 24 meses. El corte de espesor de CFNR se basó en los valores de referencia ajustados por edad (los valores < quinto percentil se consideró por debajo de los límites normales).
Imagen por resonancia magnética (IRM) de cerebro

En todos los pacientes se realizó IRM de cerebro con gadolinio con todas las secuencias, al inicio, a los 12 meses y al final (24 meses) del estudio, utilizando un equipo MARCA Phillips de 3.0 Tesla.

\section{Análisis estadístico}

Para el análisis de los resultados obtenidos se utilizó el programa estadístico SPSS versión 15.0 para Windows (SPSS Inc., Chicago, IL, USA). Para el análisis de las correlaciones entre las variables se utilizó regresión lineal con $\mathrm{R}$ de Pearson y t de Student.

\section{RESULTADOS}

El sexo femenino fue el más frecuente con $66.1 \%$. La mediana de edad fue de 33 años, el tiempo de diagnóstico promedio fue 3.21 años. De los 59 pacientes (118 ojos) iniciales, 17 pacientes (34 ojos) salieron durante el transcurso del estudio por cumplir criterios de eliminación. La EDSS basal más alta fue de 5.5 y la más baja de 0 , a los 24 meses la más alta fue de 8 y la más baja continuaba siendo 0 .

Todos los pacientes se dividieron en dos grandes grupos: el primer grupo de EM con historia de NO (EM-NO) y el segundo grupo de EM sin historia de NO (EM-NON); 38 ojos (32.20\%) tenían historia de alteraciones visuales y/o NO correspondientes a 22 pacientes (37.22\%), de los cuales nueve $(15.2 \%)$ presentaban $\mathrm{NO}$ bilateral. De las NO unilaterales el lado más afectado fue el ojo izquierdo (OI) en 57.8\%. 67.80\% (80 ojos) no tenían historia de NO (EM-NON).

Con respecto a los PEVs la onda P-100 con la latencia más prolongada estuvo en 177 ms y tres ojos no mostraban respuesta. En la OCT el espesor promedio de CFNR tanto global (G-CFNR) como de cuadrante temporal (T-CFNR) se realizó en todos los pacientes. El espesor promedio G-CFNR más bajo lo presentó un OD con 57 micras y el espesor promedio T-CNFR más bajo estuvo en 34 micras también de OD. La disminución más significativa de micras en la OCT final con respecto a la basal fue de -62 micras correspondiente a un paciente que presentó brotes visuales al sexto y doceavo mes de iniciado el estudio, la mínima disminución promedio de CFNR en la OCT final con respecto a la basal fue de -3 en un paciente que mostró mejoría en la EDSS a los 24 meses.

Considerando los 118 ojos del inicio del estudio a los cuales se realizaron PEVs, 56.78\% (67 ojos) presentaban PEVs anormales, de los cuales $50.75 \%$ eran OI. Por otro lado, 50.85\% de las OCT mostraban valores disminuidos, de los cuales $51.66 \%$ correspondían al OI. Ambos estudios 
fueron anormales en 53.39\% (63 ojos). Aunque 21.2\% (25 ojos) presentaron PEVs anormales con OCT en valores normales. $11.9 \%$ (14 ojos) mostraron valores G-CFNR disminuidos con PEVs normales.

\section{PEVs y OCT}

En el grupo de pacientes con EM-NO (38 ojos), los PEVs estuvieron anormales en $94.7 \%$, por otro lado, la OCT estuvo en rangos anormales en $78.95 \%$ de los ojos. Solamente $2.63 \%$ (un ojo) se encontraba con PEVs normal en presencia de valores G-CFNR disminuidos; sin embargo, 23.7\% (nueve ojos) presentaron PEVs anormales con espesor G-CFNR en valores normales. Ambos estudios fueron anormales en $73.7 \%$ (20 ojos) de este grupo de pacientes.

Por otro lado, en el grupo de pacientes con EM-NON (80 ojos), 36.3\% (29 ojos) presentaron PEVs anormales, mientras que el espesor G-CFNR estuvo disminuido en 31.3\% (25 ojos). $17.5 \%$ (14 ojos) mostraron PEVs anormales con espesor G-CFNR en límites normales, mientras que $16.25 \%$ (13 ojos) presentaron valores G-CFNR disminuidos con PEVs en límites normales. Ambos estudios estuvieron normales en $80 \%$ (64 ojos).

\section{EDSS y actividad de la enfermedad}

Hubo 26 pacientes (44.0\%) que presentaron datos de actividad de la enfermedad (definida por actividad radiológica por mayor número de lesiones en T2 y Flair y/o lesiones nuevas que refuerzan con gadolinio y/o brote clínico por nuevo déficit focal neurológico) en algún momento durante los 24 meses de seguimiento, de los cuales 11\% (siete) correspondieron a neuritis óptica durante su seguimiento. $45.23 \%$ presentaron un aumento promedio de EDSS basal de uno a 24 meses. 38.09\% mostró una disminución de la EDSS basal promediada de 0.5 .

\section{DISCUSIÓN}

Durante la interpretación de los resultados del presente estudio se correlacionaron los PEVs y la OCT con la clínica, la función visual, la escala del estado de discapacidad ampliada por sistema funcional (EDSS, FSS) al inicio del estudio y al final con o sin historia de alteración visual, incluyendo la diferencia en la EDSS al final del estudio y la sensibilidad de los PEVs en pacientes con esclerosis múltiple con o sin historia de neuritis óptica (NO).

En pacientes EM-NO observamos que el promedio de G-CFNR en la OCT basal estaba disminuido (OD $85.1 \pm$ 16.8; $p<0.003$, Ol $83.0 \pm 16.1 ; p<0.0001$ ), al igual que los PEVs en pacientes EM-NO se observaron con valores de latencia de la onda P-100 muy prolongadas (OD $152.9 \pm$
17.0; $p<0.0001$, Ol $151.8 \pm 19.5 ; p<0.0001$ ), lo que apoya una correlación inversa estadísticamente significativa para los PEVs por mayor prolongación de las latencias pico de la onda P-100 y disminución global del espesor de las capas de fibras nerviosas de la retina (G-CFNR) en la OCT (OD: $\mathrm{R} 0.45 ; \mathrm{p}=0.00003$. OI: $\mathrm{R} 0.52, \mathrm{p}=0.00002$ ) como se muestra en la Figura 1 A y $B$, lo que explicaría el daño axonal ocasionado por los brotes de NO, asimismo ya reportado por otros investigadores, ${ }^{11,12}$ y el aumento de la sensibilidad en pacientes EM-NO al combinar estos dos estudios.

No obstante, en pacientes EM-NON también encontramos una tendencia estadísticamente significativa hacia la anormalidad de los PEVs (OD $114.8 \pm 14.6$, OI 114.4 $\pm 15.0 ; p<0.0001$ ), lo que no se correlacionó con la OCT de EM-NON (OD $97.3 \pm 11.9$, OI $100.4 \pm 12.3$; p $<0.0003$ ), ya que en general se encontraban en valores aceptables, lo que hace a la OCT un estudio menos sensible, pero más específico.

Al correlacionar los PEVs con el sistema funcional visual de la escala del estado de discapacidad ampliada (EDSS)

Figura 1: A y B) Análisis de regresión lineal. Espesor G-CFNR OD y OI, grosor promedio de las capas de fibras nerviosas de la retina del OD, ojo derecho y del OI, ojo izquierdo. P-100, latencia de la onda P-100.

A
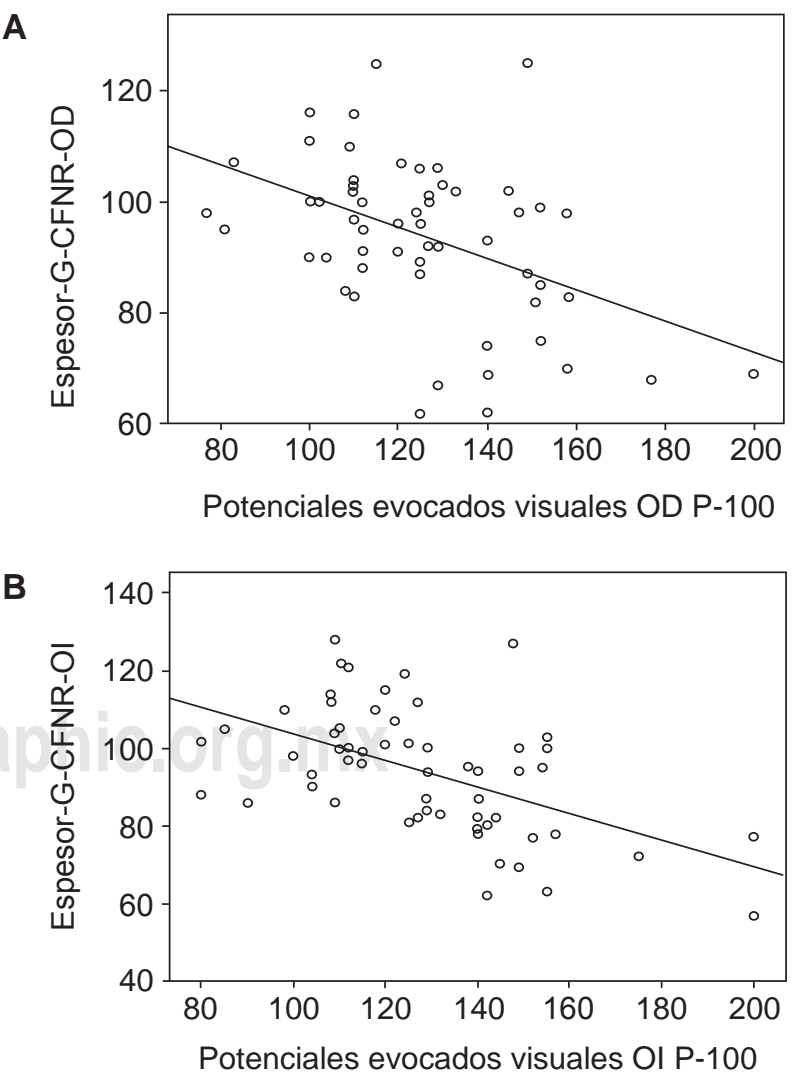
Figura 2: A y B) Análisis de regresión lineal. OD ojo derecho, OI ojo izquierdo. P-100, latencia de la onda P-100. La función visual estuvo esquematizada con base en la agudeza visual lejana medida de forma clínica, donde $0(A V=20 / 20)$ y $6(\geq 20 / 200)$.
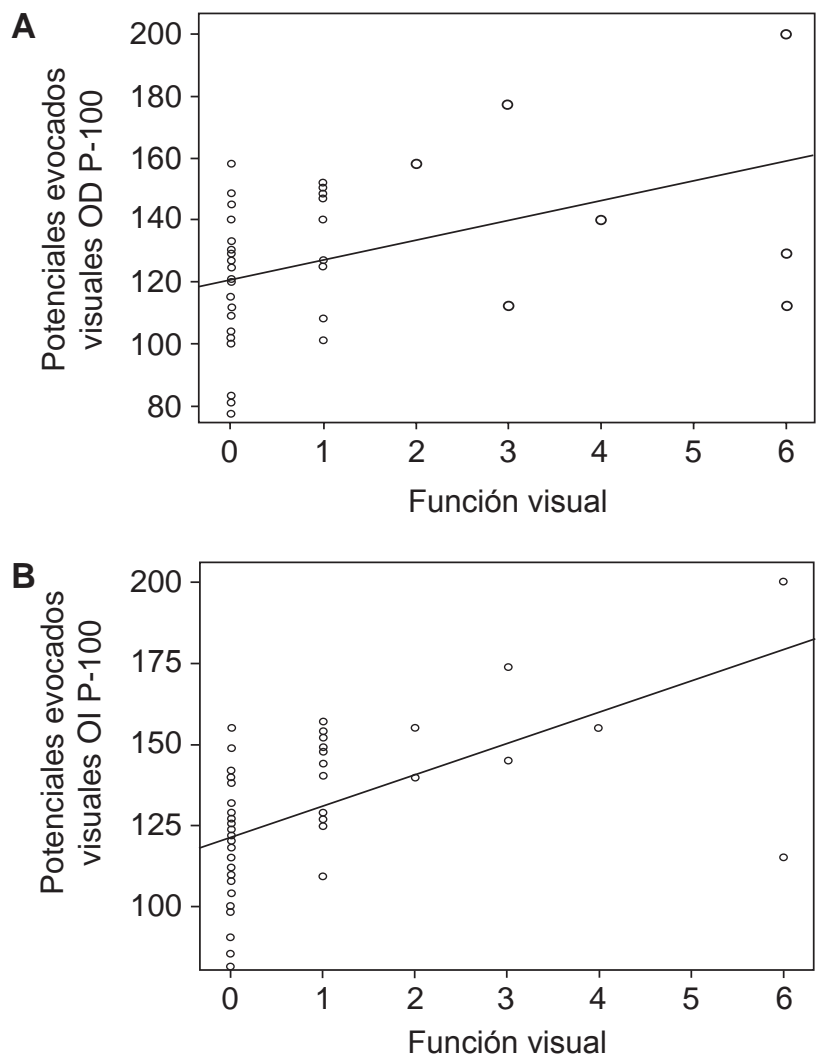

encontramos una correlación estadísticamente significativa (OD; R 0.42, p = 0.00002, Ol; R 0.60, p < 0.00001) con la prolongación de la latencia de la onda $\mathrm{P}-100$ en pacientes EM-NON y EM-NO, lo anterior se muestra en la Figura $2 \mathrm{~A}$ y $B$. Al hacer a los PEVs un estudio altamente sensible para pacientes con esclerosis múltiple con o sin alteraciones en la vía visual, representan en los pacientes EM-NON un daño axonal subclínico, hallazgo que constata lo ya documentado en estudios previos realizados por otros investigadores. ${ }^{13}$

Sin embargo, al comparar los PEVs con la EDSS final a los 24 meses no encontramos una correlación estadísticamente significativa $(\mathrm{OI} R=0.23, \mathrm{p}=0.8, \mathrm{OD} R=0.19$, $p=0.7$ ), lo que explica el hecho de que, aunque este estudio tiene una alta sensibilidad para el diagnóstico, presenta nula relevancia en el pronóstico o la evolución de la enfermedad.

Por otro lado, al cruzar los resultados de la OCT a los 24 meses y el diferencial entre la EDSS basal y final, encontramos una correlación estadísticamente significativa para el $\mathrm{OI}(\mathrm{R} 0.87, \mathrm{p}<0.00001)$ no significativa para el OD ( $R$-0.63, P 0.4). Se muestra en la Figura 3 A y B. Lo que se puede explicar por el hecho de que hubo más brotes visuales de EM-NO en Ol, contribuyendo a su vez a un empeoramiento de la escala de EDSS en dichos pacientes.

No obstante, al correlacionar los valores de G-CFNR en la OCT final con la actividad de la enfermedad durante los 24 meses, observamos una clara significancia estadística (OD; R 0.28, p < 0.0001, Ol; R 0.41, p = 0.002).

Con similares resultados al correlacionar los valores de G-CFNR en la OCT final con la función visual esquematizada por la agudeza visual y por la escala de sistemas funcionales de la EDSS al final del estudio (OD; R 0.35, p $=0.0004, \mathrm{OI} ; \mathrm{R} 0.28, \mathrm{p}<0.0001$ ).

Lo anterior evidencia que los pacientes que mostraron mayor disminución del espesor de G-CFNR en la OCT, se relacionaron con actividad de la enfermedad con respecto a los que no tuvieron dicha actividad y que los pacientes que mostraron menos disminución del espesor de G-CFNR, tuvieron mejor función visual, contrario a los que mostraron mayor

Figura 3: A y B) Análisis de regresión lineal. Espesor G-CFNR 24 meses OD y Ol; grosor promedio de las capas de fibras nerviosas de la retina de ojo derecho y ojo izquierdo. Diferencial de calificación final EDSS a los 24 meses, definido como el cambio en la puntuación de la EDSS al final del estudio con respecto a la puntuación basal.

EDSS = escala del estado de incapacidad ampliada.

\section{A}
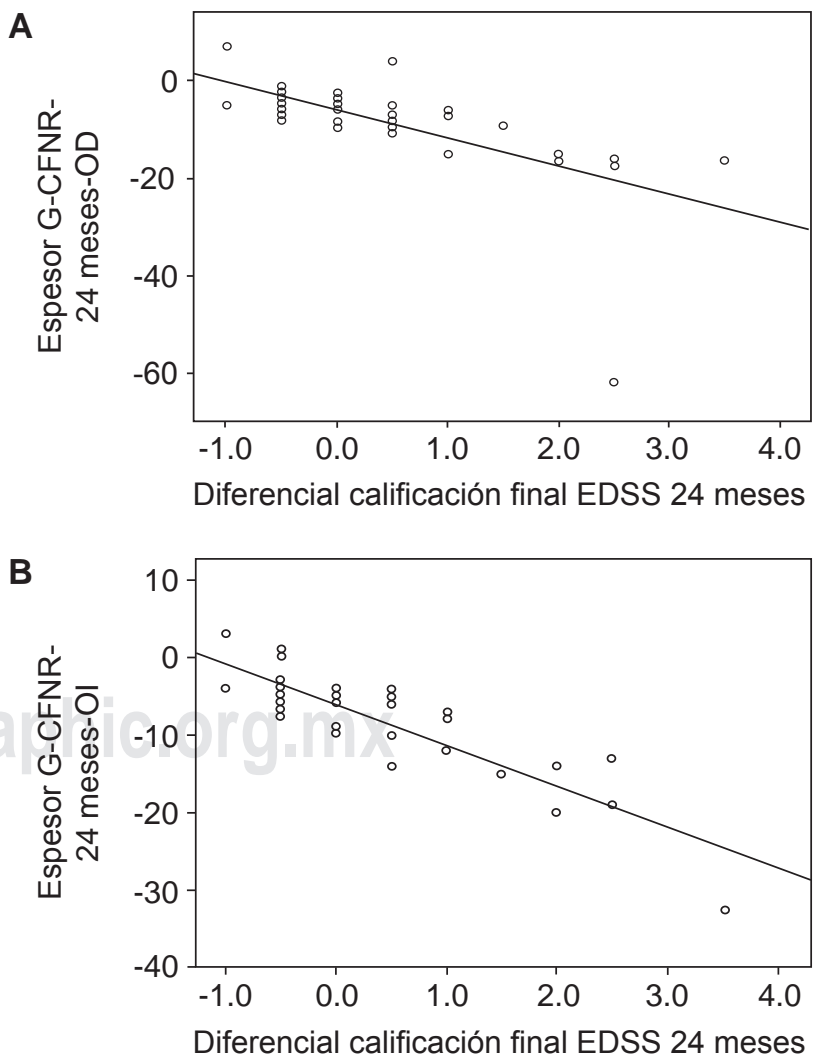
disminución del espesor de G-CFNR, datos que corresponden a lo descrito en otras literaturas sobre la atrofia de CFNR como un fenómeno precoz en la EM, incluso en pacientes que no han padecido alteraciones visuales, colocándolo como un estudio de pronóstico y seguimiento en pacientes con EM con o sin historia de NO por el daño axonal subclínico degenerativo de la enfermedad, aunque poco sensible para detectar alteraciones subclínicas y/o recientes. ${ }^{14}$

\section{CONCLUSIÓN}

La evaluación neurofisiológica y estructural de la vía visual en pacientes con EM constituye en conjunto una herramienta de gran importancia. Con estos resultados podemos determinar la correlación neurofisiológica funcional y estructural entre estos estudios y la valoración clínica, ofreciendo al paciente y al médico mayor seguridad en cuanto al estado de actividad, la evolución de la enfermedad, los tratamientos y el pronóstico de estos pacientes. Actualmente existen nuevas herramientas en esta área, con las cuales no contamos plenamente en el país, así como otras que se están desarrollando con el fin de poder limitar y precisar topográficamente de forma no invasiva la neurodegeneración que existe en esta enfermedad.

Los resultados de este estudio aportan información relevante en cuanto a la vía visual en pacientes mexicanos con EM-RR, corroboran la alta sensibilidad de los PEVs en pacientes EM-NON y EM-NO y la correlación de los PEVs-OCT entre ellos y con la clínica, la función visual y la EDSS al final. Del mismo modo, establecen un valor pronóstico de la OCT en pacientes mexicanos con EMRR con disminución del espesor de G-CFNR relacionada con aumento en la discapacidad a dos años.

\section{REFERENCIAS}

1. Fisher JB, Jacobs DA, Markowitz CE, Galetta SL, Volpe NJ, NanoSchiavi ML et al. Relation of visual function to retinal nerve fiber layer thickness in multiple sclerosis. Ophthalmology. 2006; 113: 324-332.
2. Chatziralli IP, Moschos MM, Brouzas D, Kopsidas K, Ladas ID. Evaluation of retinal nerve fiber layer thickness and visual evoked potentials in optic neuritis associated with multiple sclerosis. Clin Exp Optom. 2012; 95: 223-228.

3. Almarcegui C, Dolz I, Pueyo V, García E, Fernández FJ, Martin J et al. Correlation between functional and structural assessments of the optic nerve and retina in multiple sclerosis patients. Neurophysiol Clin. 2010; 40 (3): 129-135.

4. Soderstrom M. Optic neuritis and multiple sclerosis. Acta Ophthalmologica Scandinavica. 2001; 79: 223-227.

5. Thurtell MJ, Bala E, Yaniglos SS, Rucker JC, Peachey NS, Leigh RJ. Evaluation of optic neuropathy in multiple sclerosis using low-contrast visual evoked potentials. Neurology. 2009; 73 (22): 1849-1857.

6. Sergott RC. Optical coherent tomography: measuring in vivo axonal survival and neuroprotection in multiple sclerosis and optic neuritis. Curr Opin Ophthalmol. 2005; 16: 346-350.

7. Huang D, Swanson EA, Lin CP, Schuman JS, Stinson WG, Chang W et al. Optical coherence tomography. Science. 1991; 254 (5035): 1178-1181.

8. Costello F. Evaluating the use of optical coherence tomography in optic neuritis. Multiple Sclerosis International. 2011; 2011: 148394.

9. Toledo J, Sepulcre J, Salinas-Alaman A, García-Layana A, MurieFernandez $M$, Bejarano $B$ et al. Retinal nerve fiber layer atrophy is associated with physical and cognitive disability in multiple sclerosis. Multiple Sclerosis. 2008; 14: 906-912.

10. Gordon-Lipkin E, Chodkowski B, Reich DS, Smith SA, Pulicken M, Balcer LJ et al. Retinal nerve fiber layer is associated with brain atrophy in multiple sclerosis. Neurology. 2007; 69: 1603-1609.

11. Galetta KM, Balcer LJ. Measures of visual pathway structure and function in MS: clinical usefulness and role for MS trials. Multiple Sclerosis and Related Disorders. 2013; 2: 172-182.

12. Vladimirova Z, Shmarov A, Cherninkova S. Optical coherence tomography and its correlations with VEP in multiple sclerosis patients. Journal of Neurology and Neuroscience. 2016; 7 (6): 163.

13. Sriram P, Wang C, Yiannikas C, Garrick R, Barnett M, Parratt J et al. Relationship between optical coherence tomography and electrophysiology of the visual pathway in non-optic neuritis eyes of multiple sclerosis patients. Plos One. 2014; 9 (8): e102546.

14. Esen E, Sizmaz S, Balal M, Yar K, Demirkiran M, Unal I et al. Evaluation of the innermost retinal layers and visual evoked potentials in patients with multiple sclerosis. Curr Eye Res. 2016; 41 (10): 1353-1358.

Conflicto de intereses: Los autores declaran no tener ningún conflicto de intereses.

Financiamiento: Los autores declaran no haber recibido patrocinio para la realización del trabajo. 